\title{
CORRUPCIÓN EN LAS CONTRATACIONES DEL ESTADO: EL NEFASTO CLUB DE LA CONSTRUCCIÓN EN EL PERÚ
}

\author{
CORRUPTION IN STATE CONTRACTS: THE CONSTRUCTION CLUB NEFASTE IN \\ PERU
}

\author{
Julio Alberto Hennings Otoya \\ Universidad Nacional Mayor de San Marcos \\ Lima, Perú \\ ORCID: https://orcid.org/0000-0002-4195-5159
}

Correo electrónico: jhenningso@unmsm.edu.pe

\section{RESUMEN}

El estudio aborda la problemática de la corrupción en el sector de la construcción del Perú, centrándose en las prácticas ilegales llevadas a cabo en el caso conocido como el "Club de la Construcción", organización de directivos de empresas privadas que, utilizando prácticas corruptas y coludidos con funcionarios y altas autoridades del gobierno, logró acaparar y sobrevalorar las obras públicas licitadas por el Estado durante un lapso estimado que superaría los 20 años, con lo cual encarece el costo de los proyectos públicos y afecta la competencia del sector, entre otros graves inconvenientes. El artículo describe los hechos principales, teniendo como objetivo analizar la problemática de la corrupción en la contratación de obras del Estado peruano, reflexiona sobre sus causas y el efecto que produce en la eficiencia del mercado de dicho sector, sugiriendo recomendaciones que puedan mitigar el riesgo en tal operativa del sector estatal. En el estudio se acude a la revisión documental, teorías e hipótesis de mercado, entrevistas de expertos y el análisis de los hechos.

Palabras clave: Corrupción; colusión; libre competencia; código de ética; sector construcción.

\begin{abstract}
The study addresses the problem of corruption in the construction sector of Peru, focusing on illegal practices carried out in the case known as the "Construction Club", an organization of executives of private companies, that using corrupt practices colluded with officials and high government authorities, managed to hoard and overvalue the public works tendered by the State for an estimated period that would exceed 20 years, thereby increasing the cost of public projects and affecting the competitiveness of the sector, among other serious inconveniences. The article describes the main facts, with the objective of analyzing the problem of corruption in the contracting of works of the Peruvian State, reflecting on its causes and the effect it produces on the efficiency of the market in that sector. Suggestions and recommendations that could mitigate the risk in such operations in the State sector were offered. In the study, the documentary review, market theories and hypotheses, expert interviews and the analysis of the facts were used.
\end{abstract}

Keywords: Corruption; collusion; free competition; code of ethics; construction sector.

(c) Los autores. Este artículo es publicado por la Revista Quipukamayoc, Universidad Nacional Mayor de San Marcos. Este es un artículo de acceso abierto, distribuido bajo los términos de la Licencia Creative Commons Atribución-NoComercial-Compartirlgual 4.0 Internacional.(http://creativecommons.org/licenses/by-nc-sa/4.0/), que permite el uso no comercial, distribución y reproducción en cualquier medio, siempre que la obra original sea debidamente citadas. 


\section{INTRODUCCIÓN}

El estudio de la corrupción, sus secuelas y el riesgo de incurrir en ella, es abordado en tiempos recientes por un buen número de instituciones públicas y privadas, como organismos multilaterales, organismos no gubernamentales, órganos de control estatales, empresas consultoras e investigadores académicos de universidades de diversos países. Referente a lo pernicioso que este fenómeno tan vigente resulta, se aprecia, en distintas opiniones de entendidos que convergen, que los flujos generados por los delitos "más graves" vinculados a la corrupción podrían ascender al 20\% del PBI mundial, afectando en su paso a la economía, la política, el orden social, el comercio, el medio ambiente global, entre otros, impactando consecuentemente en las finanzas públicas, en la distribución de la riqueza generada y en la libre competencia empresarial.

Dentro de las organizaciones especializadas en el orden internacional, es de destacar una ONG que, desde su fundación en 1993, evalúa y emite iniciativas contra los delitos de las instituciones públicas y privadas, relacionadas a la corrupción, cuyo nombre es Transparencia Internacional (TI). Esta reconocida entidad, en su publicación anual Índice de Percepción de Corrupción, señala una lista de corrupción por países, donde precisamente el Perú, aparece en la posición media inferior -en el número 101 de 180 países evaluados en el año 2019, con solo 36 puntos obtenidos sobre 100 de dicho ranking. El panorama en la región tampoco es alentador, puesto que solo tres países de Latinoamérica (Uruguay, Chile y Costa Rica) cruzan la media del puntaje, y más aún, al observarse el informe total, llama a reflexión que más de dos tercios de países califiquen con menos de 50 puntos, en la escala que va desde cero (altamente corrupta) a cien (muy limpia), lo cual demuestra el alto grado de corrupción que hoy existe en el planeta. Adicionalmente, otra publicación de esta ONG remarca el peligro que produce la falta de transparencia y rendición de cuentas en la adjudicación de licencias, permisos y contratos del Estado con el sector minero, justamente en el inicio de su cadena de valor, siendo esta la causa principal de corrupción de dicho sector (Transparency International, 2018).

En el plano local, se tiene a Proética, que es el Capítulo Peruano de la anterior TI, constituida en el 2002 como ONG peruana dedicada exclusivamente a promover la ética y luchar contra la corrupción. Dentro de sus actividades, con el objetivo de erradicar la corrupción en el país, estudia el fenómeno y sus causas, e incentiva a otras instituciones a involucrarse en dicha problemática y tomar acción frente a ella. Sus miembros participan frecuentemente con entrevistas, artículos en medios periodísticos y eventos públicos buscando sensibilizar a las autoridades, empresas y público sobre los altos costos que genera la corrupción en la economía y la sociedad.

En cuanto a empresas privadas que investigan este crucial tema, se aprecia a la consultora y auditora KPMG que advierte la existencia de riesgos asociados a la Ley Anticorrupción peruana, como la ausencia de políticas y procedimientos para prevenir y detectar conductas irregulares, la administración que adolece de conocimiento de responsabilidades y legislaciones ante el cohecho y soborno, la ausencia del due diligence en empleados tercerizados, la falta o desconocimiento del manual de ética de la institución, así como controles internos débiles, entre otros. Señala, además, que las empresas pueden perder un $5 \%$ de sus ingresos anuales motivado por los fraudes (KPMG, 2016). Al respecto, merece comentar la falta de código o manual de ética en muchas de las webs oficiales tanto de empresas importantes como de sus gremios empresariales que las congregan.

Entre los trabajos académicos latinoamericanos, Gómez (2014) planteó que la corrupción está inmersa en el ámbito empresarial, y que se vincula a la vez con la colusión, por lo cual, adicional a la normativa que imponga el Estado, es preciso que las instituciones velen por evitar estas lacras en su gestión, contemplando regulaciones internas, como un Reglamento Anticorrupción alineado a los compromisos internacionales del país. En este orden de ideas, serían contraproducentes los incentivos dinerarios que otorgan los privados a funcionarios estatales, despertando su codicia, y abandonando sus responsabilidades funcionales para enriquecerse, como narra el caso de la presente investigación.

Respecto a los factores estructurales que inciden en el fenómeno de la corrupción, Estévez y Marini (2004) menciona que existen cinco que le dan forma, ellos serían la concentración del poder y riquezas junto a la impunidad, las desigualdades sociales materiales en derechos y en deberes, la racionalidad instrumental: eficientismo-pragmatismo, la caída de valores que fundamentan las decisiones públicas-tecnocracia, y el egoísmo-individualismo como razón propia. La existencia de estos factores reiterativos que también presenta la sociedad peruana se expresan en el ámbito cada vez mayor de la concentración de riqueza y la influencia que tienen los grupos empresariales de poder en los gobiernos y la sociedad, la cual soslaya la importancia de valores básicos como la sinceridad, la honestidad, la solidaridad, el compromiso social, etc., necesarios para una adecuada convivencia social; esto lo podemos intuir con la inexistencia de un código o reglamento de ética visible y transparente que incorpore tales valores, en la mayoría de empresas y sus instituciones gremiales, tal como 
se observa en la página web de la Confederación Nacional de Instituciones Empresariales Privadas-CONFIEP (2015), que agrupa a organizaciones de distintos sectores del país.

Sobre el tema de asociar la facilidad de hacer negocios con los índices de corrupción en las obras públicas, León (2013) encuentra que en la medida en que los países son más engorrosos los permisos y normas para la construcción, generan un peor índice de ética y corrupción. Comenta que los gobiernos locales que administran dichos permisos suelen tener procesos complejos que son saltados por la acción de funcionarios corruptos, invitando luego a pagos ilegales. Es decir, se podría afirmar que, a más dificultades burocráticas para el emprendimiento y los negocios, mayor será la proximidad en el riesgo de delinquir tanto por el lado del supervisor del Estado como del empresario administrado.

Respecto a la investigación central, resultó trascendente en el tema de corrupción internacional y que ha contribuido a destapar el presente caso de fuerte impacto en el Perú, la publicación a fines de diciembre del 2016, de un documento del Departamento de Justicia de Estados Unidos en el cual se reveló cómo Odebrecht (miembro importante del Club de la Construcción) operó a través de actos delictivos, como el soborno a funcionarios estatales, para conseguir le otorguen proyectos y concesiones en distintos países y seguir operando durante mucho tiempo de manera ilícita. Según dicho documento, sobre su accionar en el Perú, Odebrecht reconoció el pago de sobornos entregados a altos ejecutivos de Gobierno, sucedidos entre el año 2005 y el 2014. Esta declaración puso en alerta a la justicia peruana, abocándose a investigar las licitaciones y contrataciones de obras importantes concedidas a Odebrecht en los periodos de los exmandatarios Toledo, García y Humala, y a Pedro Pablo Kuczynski por su participación pública como exministro. El mismo texto, consigna que los ingresos de la empresa corrupta fueron de ciento cuarenta y tres millones de dólares (District Court Eastern District New York, 2016).

En la otra orilla del pensamiento respecto a la corrupción, afirmando que puede ser favorable para el crecimiento económico, se encuentran autores como Nathaniel Leff, y Samuel Huntington, ambos creyentes que la corrupción puede tener un lado positivo, como facilitar los procesos burocráticos y permitir la dinamización de la economía, en cuyo ambiente las empresas se verían beneficiadas ganando contratos, lo que generaría a la sociedad, más puestos de trabajo y rentas para el consumo (Huntington, 2014; Leff, 2002).

En una posición menos complaciente frente a la corrupción, Gómez-Jara (2012) sugiere:
Normar la responsabilidad legal de los administrados, que resultarían las empresas privadas, pero $l i$ mitando su responsabilidad de la corrupción solo a lo que concierne a la competencia desleal, relegando la infracción de los deberes cometidos a la constatación de la existencia o no, del delito que subyace de su administración desleal. (p.225)

Frente a los antecedentes expuestos, con investigadores de diferente enfoque y énfasis en las consecuencias de la corrupción, surge la interrogante que servirá de guía al presente artículo: ¿La corrupción vinculada a la contratación de obras del Estado peruano afecta a la competencia del sector?

Las razones por las que se abordó la temática del riesgo de la corrupción en las contrataciones del Estado están vinculadas a la necesidad de conocer con mayor detalle dicho problema que ha venido generando grandes pérdidas al país, tanto de naturaleza tangible a través de costos excesivos detectados en las obras, como de naturaleza intangible con la pérdida de confianza en las instituciones que deberían participar en los procesos y la desmotivación de la población trabajadora. Superar ambos aspectos, resultaría fundamental en la mejora competitiva para encaminarnos en las metas del desarrollo, con connotación económica y social.

Por otro lado, relacionar el flagelo de la corrupción en la contratación de obras que enfrenta la realidad nacional tanto por las instituciones públicas como por las privadas, cuyo nefasto caso se muestra con el club de la construcción, es buscar respuesta al porqué de la debilidad o inexistencia de competencia en ese sector, aspecto clave para que funcione con eficiencia la economía social de mercado que prescribe la Constitución Política del Perú.

En cuanto a las limitaciones del estudio, es de mencionar que como acontecimiento social que se presenta en un tiempo tan extenso, el presente trabajo podría haber recogido solo los principales hechos y que fueron documentados, ya que los otros probables, quedaran solo en la mente y los recuerdos de los involucrados, en uno de los casos más notorios de corrupción destapados en el país.

\section{Metodología de trabajo}

El diseño de la investigación es no experimental, con alcance descriptivo y explicativo, siendo los métodos principales utilizados el analítico y el deductivo. En el desarrollo del trabajo, se contrastó la información obtenida de distintas fuentes (primarias y secundarias); y en cuanto a técnicas de estudio, se utilizó la revisión documental, la técnica comparativa y entrevistas de expertos del sector privado y público. 


\section{La corrupción privada y el clima de negocios}

Generalmente, las críticas y medidas que se esgrimen para combatir la corrupción van direccionadas al ámbito estatal; sin embargo, se tiene que reflexionar también como señala Gómez (2014) que:

El sector privado tiene mucha injerencia en los altos niveles de corrupción...En el día a día se piensa que sólo los políticos son corruptos, pero el tema del soborno es algo crítico en el sector privado al igual que la financiación política. (Como se citó en Ungar, 2011, p. 45)

Sin embargo, como bien señala Lazarte (2018) "no existe fórmula mágica para combatir lo enraizado de la corrupción en el país, ni el ejecutivo, ni el parlamento, ni el poder judicial podrán afrontarlos solos porque el problema no es político, ni legal, es social". Es decir, que, ante la ausencia de valores, la corrupción se muestra solo como uno de sus síntomas, por lo que es necesario que el sector privado contribuya con la solución, cual es de impartir y fijar los valores necesarios en la sociedad, a través de sus medios.

Un factor que facilita llevar a cabo las coimas de la corupción es la difícil tramitación de obras y permisos que muchos países tienen para el sector constructivo, lo cual al derivar en engorroso (y costoso) el proceso, algunas empresas privadas del sector optan por superarlo con "incentivos" al controlador del trámite, lo cual ven como una salida que les permitirá agilizar actividades y hacer factible las ganancias del negocio.

Sobre el tema, la publicación del World Bank, International Finance Corporation \& Palgrave MacMillan (2009) comentan que en ningún país resulta sencillo que los gobiernos simplifiquen sus trámites, lo cual implica mayor coordinación entre los empresarios y los gobiernos para armonizar reglas a favor del clima de negocios.

Como se aprecia en la figura 1, el ranking relativo de facilidad de hacer negocios está muy vinculado al ranking de trámites en la construcción; en la medida que los países son más engorrosos en estos rubros, generan peor performance en el índice de ética y corrupción.

\section{Colusión y la libre competencia}

Citando el diccionario de la Real Academia de España, el vocablo colusión es el pacto ilícito en detrimento de tercero y como vocablo tiene su origen en la expresión latina collusio-collusionis que se deriva del verbo colludo-colludere que significa "jugar juntos" (Sierra, 2003). En extensión a lo anterior, colusión se utiliza para referir la sinergia fraudulenta de complicidad de los integrantes o participantes en un proceso de selección para obras del

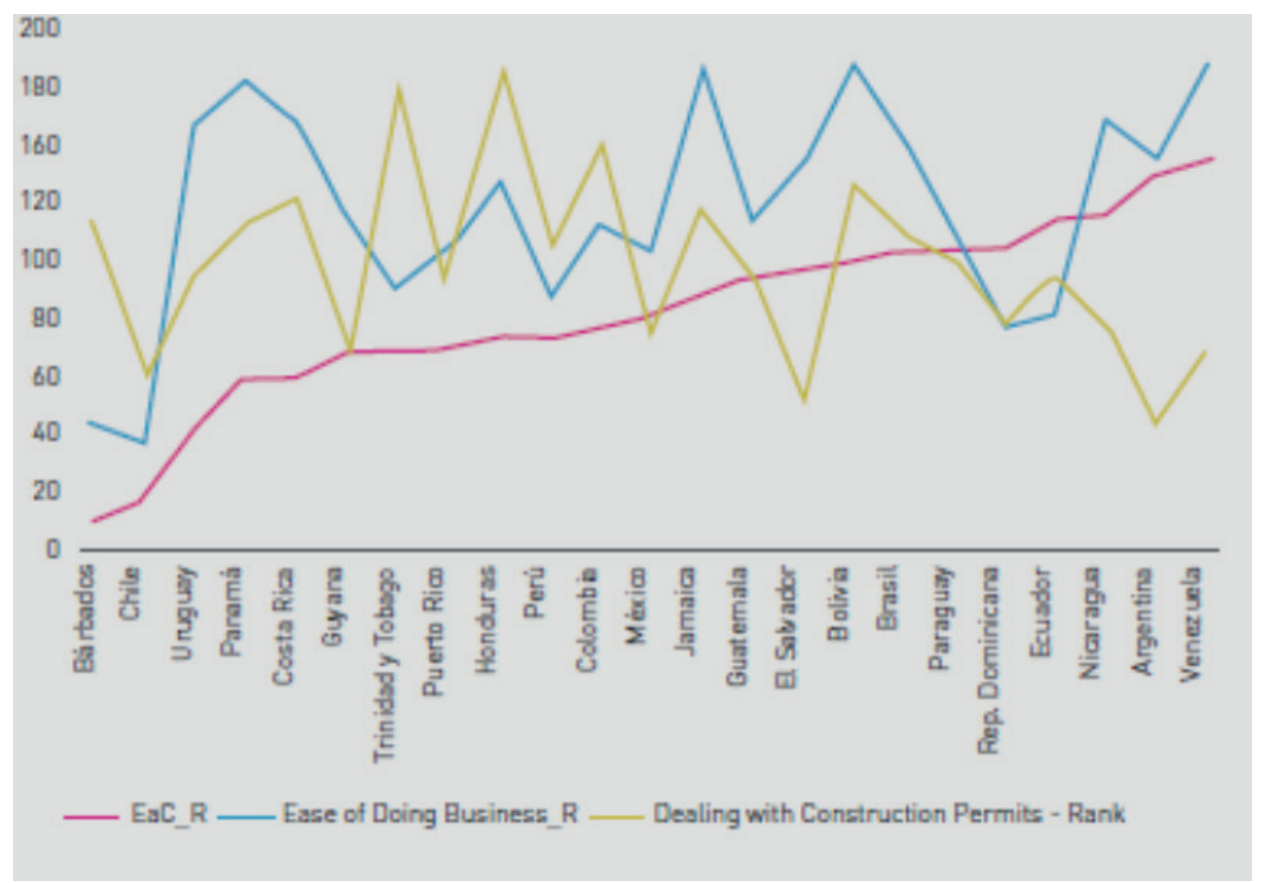

Figura 1. Relación entre índice de ética y corrupción, facilidad de hacer negocios y permisos de construcción, el indicador más alto es el de peor desempeño en la región.

Fuente: Foro Económico Mundial (WEF por sus siglas en ingles), 2011. 
Estado, afectando derechos o en detrimento de terceros, para competir en condiciones de igualdad. En tal sentido, la colusión dañaría la libertad de empresa afectando el derecho a la libre competencia, al existir entre ambos necesariamente un vínculo conector.

En una economía en democracia, la libertad de empresa se complementa con la propiedad privada, constituyéndose en el centro de los derechos económicos en donde la libertad de empresa confrontará dos elementos típicos de la democracia, la libertad y la igualdad. Así, un sistema democrático que prioriza la libertad frente a la igualdad favorecerá sin proponérselo a los emprendedores más competentes, quienes al aventajar a los otros logran disfrutar de su posición de mercado, pudiendo generar distorsiones negativas como los monopolios o los oligopolios; y si el sistema prioriza la igualdad, utilizando el poder del Estado, coactará la libertad de quienes en base a su mayor competencia usan el mercado a su favor, y en la intención de brindar igualdad de posibilidades, obtendrá resultados con limitado talento empresarial. Ante esta disyuntiva, surgen alternativas de mercado con características intermedias como las de libertad vigilada, la regulada y la responsable. Sin embargo, tales fórmulas, al parecer, no habrían cubierto aún las expectativas de solución.

En general, estando vetado el monopolio, los oligopolios actúan dominando sus mercados, y buscan a través de aproximaciones interesadas con los gobiernos de turno reforzar sus ventajas, incrementando su dominio del mercado frente a los competidores menos fuertes, limitándoles su campo de acción y sus posibilidades de ganancias. Por otro lado, la competencia justa y abierta, normalmente estimula la innovación y la eficiencia, esperando que se reduzcan los costos de los bienes y servicios, y permite que las empresas tengan las mismas oportunidades. Así, se impulsará la mejora de productos y procesos, y con esto, se contribuirá con el crecimiento económico y el nivel de vida de la sociedad. Lo opuesto a este tipo de competencia produce fallas de mercado como la concertación de precios, las compras amañadas y la colusión, lo que precisamente determinó la existencia del Club de la Construcción.

\section{La coordinación pública privada y las puertas giratorias}

La puerta giratoria, traducida de la expresión en inglés revolving door, en lenguaje coloquial, refiere al hecho de que un funcionario público se marche a laborar a una empresa privada, obteniendo beneficio de su anterior puesto de trabajo, generándose conflictos de interés entre la esfera pública y la privada, en beneficio propio y en perjuicio del interés público. Viceversa a lo anterior, la puerta giratoria también se aplica cuando ejecutivos de poder decisorio de empresas privadas pasan a desempeñar altos cargos públicos, manteniendo muchas veces una cercanía laboral o social con sus anteriores colegas del sector privado. Estos lógicamente podrán influenciar en la toma de decisiones que competan a su labor, y en numerosas ocasiones, estos mismos funcionarios nuevamente retornan a la escena privada, favoreciéndose de los conocimientos y contactos establecidos para obtener beneficios personales en desmedro del interés público.

En el plano local, un proceso conector de intereses público-privado se da en las compras del Estado, donde a veces falta la transparencia y el accountability (rendición de cuentas) en la adjudicación de licitaciones y concursos de obras públicas; y, posteriormente, en la confección de los contratos y su seguimiento operativo, han permitido la grave corrupción suscitada en el sector construcción. Más aún, juega un rol complaciente facilitador de muchos de los delitos expuestos, las puertas giratorias en la que han operado funcionarios claves de extracción empresarial —en puestos políticos de confianza- permitiéndoles mantener sus contactos sociales y económicos para "coordinar" la obtención de beneficios indebidos afectando el interés del Estado.

De otro lado, las débiles iniciativas para mejorar la gobernanza y prevenir la corrupción en las obras licitadas, a pesar del reiterado discurso político de los últimos 20 años, han sido poco efectivas y han estado centradas en los esfuerzos de la Contraloría de la República, que con limitados recursos en sus órganos descentralizados (Oficinas de Control Interno), no ha sido capaz de neutralizar los riesgos latentes en los procesos de adjudicaciones del Estado, y en consecuencia, se han generado vacíos, que aprovechados por las organizaciones delictivas con la complicidad de algunas autoridades públicas, han socavado el otorgamiento lícito, conforme y ético de las contrataciones públicas de obras, alterando a la vez las posibilidades de una sana competencia en el sector.

De los muchos ejemplos que podemos anotar sobre las puertas giratorias en el caso "Club de Construcción" y la facilidad que otorgaban para operar sus delitos, comentamos el que se da a conocer que un nuevo colaborador eficaz del caso señaló a los fiscales, que José Paredes - hermano del exministro de Transportes, Carlos Paredes, exgerente de empresas privadas- entregó coimas a Ollanta Humala en pleno Palacio de Gobierno. La primera habría sido por 500 mil dólares el 21 de noviembre del 2011, año en el que asumió el gobierno. Además, afirmó que el hermano del exministro recibió dinero de las constructoras miembros de este club entre el 2011 y el 2014, estimando las coimas entre 16 y 18 millones de dólares.

\section{Descubrimiento del Club de la Construcción}

Para el descubrimiento del nefasto Club de la Construcción, resultó fundamental la publicación del informe del 
Departamento de Justicia Norteamericano a fines del 2016 que reveló cómo Odebrecht pudo corromper vía elevadas "comisiones" a funcionarios públicos de todo el mundo, para acaparar obras y concesiones públicas bajo características delictivas. Sobre su accionar en el Perú, Odebrecht reconoció inicialmente que el pago de sobornos había ascendido a la suma de US\$29 millones y que estuvo destinada a los funcionarios del Gobierno peruano durante el periodo de los años 2005 y 2014. Esta etapa de tiempo, que involucró a los gobiernos de Alejandro Toledo (20012006), Alan García (2006-2011) y Ollanta Humala (20112016), generó secuelas posteriores en el ámbito político de entonces, dados los descubrimientos que Odebrecht había favorecido también a Pedro Pablo Kuczynski, presidente en funciones en esa época (2016-2017), generándose una crisis presidencial y su posterior renuncia al cargo.

\section{Operativa del Club de la Construcción}

A través de los colaboradores de la fiscalía peruana, se pudo conocer que el Club de la Construcción lo integraban 30 empresas constructoras del sector privado, casi todas pertenecientes al importante gremio empresarial de la Cámara Peruana de la Construcción (CAPECO). En este gremio tenían un rol especial el investigado Rodolfo Prialé, quien fungía de representante de las empresas, y el funcionario Carlos García, quien habría recibido el 2,92\% de cada obra adjudicada, para ser repartida a los demás funcionarios corruptos del Ministerio de Transportes y Comunicaciones. Las empresas involucradas en el delito que fueron delatadas se muestran en la Figura 2.

Lazarte (2018), citando a la Fiscalía como fuente, detalla cómo Prialé entregaba el 2,92\% del valor referencial

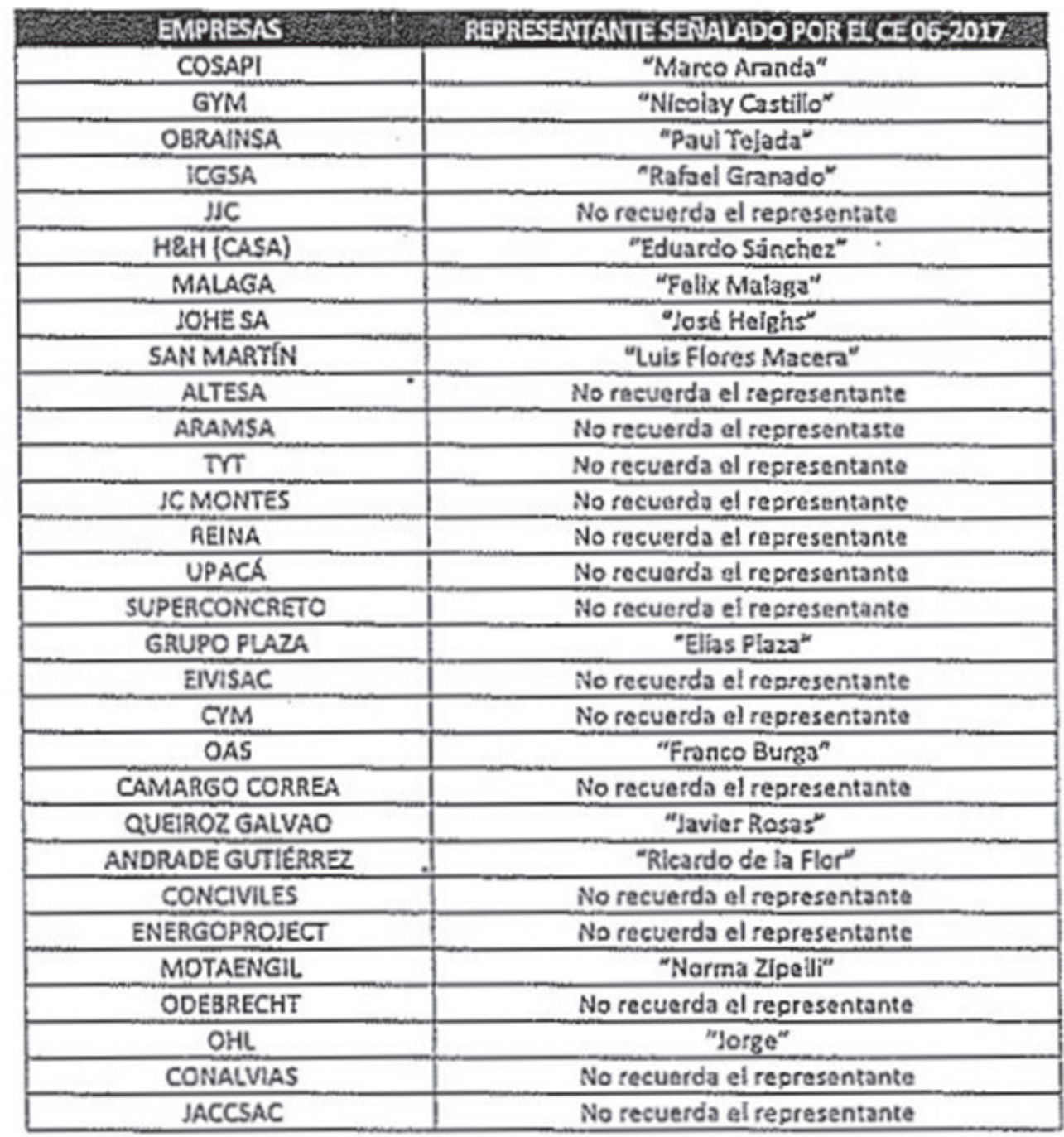

Figura 2. Empresas conformantes del Club de la Construcción y Representantes Fuente: Semana Económica 18/01/2018 (Origen mencionado: Fiscalía de la Nación) 
de las obras que eran adjudicadas a las instituciones que representaba, en manos del exfuncionario García, precisando a su vez, que actuaba como encargado de comunicar las decisiones del club a dicho exfuncionario del Ministerio de Transportes y Comunicaciones (MTC). Según la resolución del caso, las empresas acordaban qué consorcio ganaría la licitación de las obras. Dichos acuerdos previamente habían sido adoptados en las reuniones eventuales realizadas en función de los procesos de licitación. Comenta también, que la investigación ha encontrado que desde el 2010 existía una relación cercana entre el exfuncionario Carlos García y el empresario Rodolfo Prialé; todo lo cual refuerza empíricamente lo afirmado en el acápite referido a "La coordinación pública privada y las puertas giratorias".

\section{CONCLUSIONES}

A pesar de la normativa existente, la falta de transparencia y rendición de cuentas de las instituciones de gobierno en el otorgamiento y gestión de las licitaciones y concursos para realizar obras públicas en el sector de la construcción, se anida la raíz de la corrupción para la construcción de obras promovidas por el Estado, estos inconvenientes, aunados a una débil convicción de valores y al personal poco comprometido con su institución, actúa como caldo de cultivo para que fácilmente se cometan actos ilícitos en el avance de la infraestructura pública en el Perú. La oportunidad de más ingresos por la captura de las obras demandadas (sector privado - constructores), encuentra la complicidad impropia en los gestores del ofertante de dichos trabajos del país (sector público - funcionarios).

El problema subyacente de la corrupción está en la falta de valores consistentes de nuestra sociedad, que actúa como un cáncer que se ha dejado avanzar demasiado y la corrupción es solamente uno de sus síntomas. Sanar de esta enfermedad será un proceso largo y doloroso, cuyo costo se da en los años de no haber reconocido y valorado esta situación. Frente al flagelo de la corrupción, lo más importante es reconocer que la cura no está solo en manos del Estado. Los gobernantes y autoridades de los poderes del Estado, solos no sacarán al Perú de este problema, porque no tienen la capacidad de cambiar la sociedad. El problema no es político ni legal, es fundamentalmente social y si no hay involucramiento de todos, el mal podría seguir avanzando.

\section{REFERENCIAS}

CONFIEP (2015). Códigos de conducta de los gremios asociados a Confiep. Recuperado de: https://www.confiep.org.pe/pdfs/confiep-codigos-de-conducta-gremios-2015.pdf
District Court Eastern District New York. (2016). Information Cr. $N^{\circ} 16-643$ RJD. T.18 U.S.C.371 and 3551 et seq. United States of America against Odebrecht S.A. Defendant. U.S.A.

Estévez, A., y Marini, G. (2004). Corrupción y políticas públicas: Algunos factores estructurantes. Centro de investigación en administración pública,3(1), 19 pp. Recuperado de: https://www.academia.edu/6966202/ CORRUPCI\%C3\% 93 N_Y_POL\%C3\% 8DTI CAS_P\%C3\%9ABLICAS_ALGUNOS_FACTORES_ ESTRUCTURANTES_APUNTES_PARA_UNA_ AGENDA_DE_INVESTIGACI\%C3\%93N

Gómez, D. (2014). Corrupción y colusión: asuntos del sector empresarial en Colombia. Revista Prolegómenos Derechos y Valores, 43 - 56.

Gómez-Jara, C. (2012). Corrupción en el sector privado: ¿competencia desleal y/o administración desleal? Revista Icade. Revista de las Facultades de Derecho y Ciencias Económicas y Empresariales, (74), 225-243. Recuperado a partir de https://revistas.comillas.edu/ index.php/revistaicade/article/view/355

Huntington, S. (2014). El orden político en las sociedades en cambio. Barcelona: Paidós.

KPMG Perú. (2016). Nueve riesgos asociados a los delitos de corrupción en las organizaciones. Recuperado de: https://home.kpmg.com/pe/es/home/media/ press-releases/2016/11/9-riesgos-asociados-a-los-delitos-de-corrupcion-en-las-organizac.html

Lazarte, J. (2018). El rol empresarial contra la corrupción. Revista Semana Económica. Recuperado de: http://semanaeconomica.com/destrabe-empresarial/2018/07/20/soluciones-empresariales-contra-la-corrupcion/

León, C. (2013). Eficiencia pública, competencia, corrupción y facilidad de hacer negocios: Una comparación relativa para países de Latinoamérica. Recuperado de: http://revistas.uss.edu.pe/index.php/tzh/article/ view/46

Sierra, J. (2003). Diccionario Jurídico. Librería Jurídica Sánchez R. Ltda. Cuarta Edición, Bogotá.

Transparency International. (2018). Herramienta de evaluación de riesgo de corrupción de premios de minería. Recuperado de: https://www.transparency.org/news/pressrelease/ riesgos_de_corrupcion_y_formas_para_combatirlos_ concesiones_mineras 
Transparency International. (2018). Indice de percepción de la corrupción 2017. Recuperado de: https://www. transparency.org/news/feature/corruption_perceptions_index_2017
World Bank, International Finance Corporation \& Palgrave MacMillan. (2009). Doing Business 2010. Reforming through Difficult Times. DOI: 10.1596/978-08213-7961-5 